\title{
Postseizure aphasia in Wernicke's encephalopathy: a case report and review of literature
}

This article was published in the following Dove Press journal:

Neuropsychiatric Disease and Treatment

\author{
Sandhya Devaraj' \\ Sara A Muralles' \\ Chandler E Gill ${ }^{2}$ \\ Edwin Meresh ${ }^{3}$ \\ 'Loyola Stritch School of \\ Medicine, Maywood, IL 60I53, \\ USA; ${ }^{2}$ Department of Neurology, \\ Loyola University Medical \\ Center, Maywood, IL 60I53, USA; \\ ${ }^{3}$ Department of Psychiatry, \\ Consultation-Liaison Psychiatry, \\ Loyola University Medical Center, \\ Maywood, IL 60I53, USA
}

Correspondence: Edwin Meresh

Department of Psychiatry,

Consultation-Liaison Psychiatry, Loyola University Medical Center, Maywood, IL 60I53, USA

Email emeresh@lumc.edu

\begin{abstract}
This case discusses the course of a woman with a history of epilepsy, alcohol use disorder, herpes simplex virus (HSV) encephalitis, and Wernicke encephalopathy (WE) who presented with altered mental status following approximately 48 hours of vomiting. After experiencing a tonic-clonic seizure in the emergency department, she developed a fluent aphasia. Aphasias are ordinarily attributed to structural changes in the brain parenchyma, often from stroke, neoplasm, or infection. When the magnetic resonance imaging of brain failed to show changes that could explain her fluent aphasia, the neurology team consulted psychiatry to workup psychogenic aphasia. During an admission 9 months earlier, she had been diagnosed with HSV encephalitis and possible WE. There was a high degree of suspicion for recurrent HSV infection, intermittent focal seizure activity, postictal psychosis, pseudobulbar affect, or a vascular cause of her fluent aphasia. After 3 days of treatment with levetiracetam, high-dose intravenous thiamine, and aripiprazole, the patient's fluent aphasia reversed. The authors conclude that the patient's reversible fluent aphasia was not psychiatric in etiology but likely caused by her seizures, the result of subtherapeutic phenytoin levels; her electroencephalogram showed focal seizure activity in the temporal lobes, possibly affecting her language centers. Language-related neurological conditions, or aphasias, can mimic psychiatric conditions such as conversion disorder or psychosis. In patients with substance use disorders, the line between psychiatric and neurological conditions becomes even more difficult to distinguish. The paper also discusses how unique aspects of her medications - levetiracetam conferring neuron membrane fluidity; aripiprazole, a drug shown to halt brain atrophy in mouse models; and parenteral thiamine to address her deficiency and WE - have aided in the reversal of the fluent aphasia. Levetiracetam should be considered in WE and the rare occurrence of aphasia after seizures.
\end{abstract}

Keywords: seizure, aphasia, alcohol withdrawal, Wernicke's encephalopathy, levetiracetam, thiamine

\section{Introduction}

Language-related neurological conditions, or aphasias, can mimic psychiatric conditions such as conversion disorder or psychosis. In patients with substance use disorders, the line between psychiatric and neurological conditions becomes even more difficult to distinguish. Patients in alcohol withdrawal could manifest a wide range of presentation starting with initial withdrawal symptoms during the first 2-3 days and then leading to complications including delirium tremens, Wernicke's encephalopathy (WE), or hepatic encephalopathy (HE). Hallucinations are more prominent than confusion in delirium tremens, while confusion is prominent in HE. Fluent aphasia typically is not present in these three complications. A feared end-stage presentation for chronic alcoholics is Korsakoff syndrome, described by Victor et al ${ }^{1}$ as "an abnormal mental state in which memory and learning are affected out of all proportion to other cognitive 
functions in an otherwise alert and responsive patient." Prior to this irreversible state however, is the acute and reversible presentation of WE caused by thiamine deficiency. It should be noted that WE, while more easily recognized in alcohol abuse, can also be caused by anorexia nervosa ${ }^{2}$ and bariatric surgery. ${ }^{3}$ Classically, WE is characterized as ataxia, confusion, and nystagmus. An alternative clinical definition considers WE if two of the following conditions are met: dietary deficiencies, oculomotor dysfunction, and either altered mental status or mild memory dysfunction. ${ }^{4}$ In this case, we will examine the course of a patient with a long history of alcohol abuse and seizure disorder who presented with WE symptoms in addition to a fluent aphasia. She experienced a reversal of her acute aphasia under our care; to our knowledge, there is only one other case report detailing such a reversal. ${ }^{5}$

\section{Case report}

A 52-year-old right-handed woman, who we will call RR, presented to an academic medical center with multiple seizures. She had a history of alcohol abuse, liver cirrhosis, and epilepsy for which she took phenytoin; she had not had any seizures for several years. Additionally, she was treated for herpes simplex virus (HSV) encephalitis 9 months prior. Initially, RR presented with nausea and vomiting for 48 hours, likely from alcohol withdrawal. Her boyfriend found her at home, appearing confused, and witnessed her have a generalized tonic-clonic seizure. She was then brought by ambulance to the emergency department (ED) where she had another seizure (tonic-clonic movements, right $>$ left, with eyes deviated to the left and not responding). Her medications included aspirin, hydrocodone-acetaminophen, folic acid, thiamine, pyridoxine, and phenytoin $100 \mathrm{mg}$ daily. She typically drank 2-6 beers a day. Prior to the admission for encephalitis, she was seen in the ER 1 year before for alcohol abuse and fall. After the admission for encephalitis and prior to this current episode, she was seen in ER four times, all for falls and related fractures. As the patient has been lost to follow-up, we are unable to confirm the patient's consent for publication of this report. All data has been sufficiently anonymized not to cause harm to the patient or her family.

Vital signs at arrival-blood pressure 113/72, heart rate 106 , temperature $102^{\circ} \mathrm{F}\left(38.9^{\circ} \mathrm{C}\right)$, Respiratory rate 16 , body mass index (BMI) $23.77 \mathrm{~kg} / \mathrm{m}^{2}$. In the ED, RR was given $4 \mathrm{mg}$ of lorazepam, $2 \mathrm{mg}$ of intravenous (IV) diazepam and fosphenytoin $15 \mathrm{PE}$ units $/ \mathrm{kg}$. She remained unresponsive to verbal prompts with rhythmic beating of her left foot, prompting a neurology consult. Lacosamide was added and one dose of IV acyclovir was administered for empiric recurrent HSV encephalitis. Initially unresponsive on neurological examination, she slowly started following commands but became fluently aphasic. The differential diagnoses at this point included seizures with concern for status epilepticus, subtherapeutic medication, alcohol withdrawal, electrolyte derangement, and infection.

\section{Laboratory results}

RR's laboratory results were reported as follows: 1. cerebrospinal fluid: negative for infection or inflammation, PCR negative. 2. complete blood count: increased red blood cell distribution width 21.2 (normal 11\%-15.0\%), decreased platelets $101(150-400 \mathrm{~K} / \mu \mathrm{L})$. 3. complete metabolic panel: decreased potassium 2.8 (3.3-5.1), elevated anion gap 25 (4-16) decreased blood urea nitrogen 4 (7-22 mg/ $\mathrm{dL})$, increased glucose $228(70-100 \mathrm{mg} / \mathrm{dL})$, alkaline phosphatase 167 (30-110 IU/L), AST 125 (10-40 IU/L). 4. blood: lactate $3.4(0.9-1.7 \mathrm{mM} / \mathrm{L})$. 5. urine/drug: urinalysis was negative for urinary tract infection. Urine drug screen and blood alcohol were negative, and phenytoin levels subtherapeutic 4.2. 6. electrocardiogram: sinus tachycardia. 7. imaging: chest X-ray and computed tomography-head were both negative. The magnetic resonance imaging (MRI) was inconclusive, showing less prominent/no definite asymmetric flair abnormality in the left medial temporal lobe compared to her admission for HSV encephalitis 9 months prior.

RR was transferred to the neuro intensive care unit and placed on alcohol withdrawal protocol (Clinical Institute Withdrawal Assessment for alcohol; CIWA). video electroencephalogram (vEEG) did not reveal spikes or epileptiform activity (although this was after medication loading and gaze deviation resolution) and she had continued fluent aphasia. On day 2 , the cranial nerve exam showed diminished blink to threat on the right, bilateral nystagmus on horizontal gaze with a left gaze preference, and gait that was found to be impaired, requiring assistance.

Phenytoin and lacosamide were replaced with levetiracetam $750 \mathrm{mg}$ twice a day (BID) (Table 1). RR became agitated and was given haloperidol $2 \mathrm{mg}$ IV. Minutes later, she was found to be awake, with an unremarkable cranial nerve exam and without nystagmus, but intermittent fluent aphasia continued. Continuous 17-hour video EEG monitoring was performed. Video EEG (Figure 1) while aphasic showed focal slowing over the left temporoparietal region, consistent with an underlying structural or functional lesion, but no clear epileptiform activity. 
Table I Medication administration and CIWA

\begin{tabular}{|c|c|c|}
\hline Day & Medication & CIWA score \\
\hline $\mathrm{I}$ & Phenytoin $885 \mathrm{mg} \mathrm{IV}$, lorazepam $4 \mathrm{mg}$, diazepam $2 \mathrm{mg}$, thiamine $100 \mathrm{mg}$ PO & \\
\hline 2 & $\begin{array}{l}\text { Alcohol withdrawal protocol, lacosamide IV, thiamine } 100 \mathrm{PO} \text {, levetiracetam } 750 \mathrm{mg} \text { IV, } \\
\text { levetiracetam } 750 \mathrm{mg} \text { PO, diazepam I mg IV }\end{array}$ & $17,3,3,16,5,4,3$ \\
\hline 3 & Thiamine 500 mg q8h IV, levetiracetam 750 mg BID, lorazepam 2 mg PO, haloperidol 2 mg IV & 3 \\
\hline 4 & Thiamine 500 mg q8h IV, levetiracetam 750 mg BID, aripiprazole 2.5 mg PO & 3,2 \\
\hline 5 & Thiamine $500 \mathrm{mg}$ q8h IV, levetiracetam $750 \mathrm{mg}$ BID, aripiprazole $2.5 \mathrm{mg} \mathrm{PO}$, acamprosate & $0,0,0$ \\
\hline
\end{tabular}

Abbreviations: BID, twice a day; CIWA, Clinical Institute Withdrawal Assessment for alcohol; IV, intravenous; PO, per os; q8h, every 8 hours.

At this point, psychiatry was consulted for possible psychogenic aphasia (conversion disorder). On exam, the patient was neither able to sustain attention nor follow commands and presented with fluent aphasia, repeating the phrase "It was as big as your nose, from your chin to your ear... as big as your nose." Psychiatry recommended increasing thiamine from 100 per os (PO) to $500 \mathrm{mg}$ q $8 \mathrm{~h}$ IV and adding aripiprazole $2.5 \mathrm{mg}$ PO for delirium. On day 4 , mental status exam showed intermittent fluent aphasia with perseveration, as well as signs of disinhibition with episodes of inappropriate laughter. By the end of the day the patient could say, "I feel much better, I want to go home tomorrow." Transient ischemic attack workup, including an electrocardiogram with Doppler, computed tomography angiogram, and fasting lipid panel, came back negative. On day 5 , the patient sustained a 30 -minute conversation with few paraphasic errors, but frequently giggled, continuing to show signs of disinhibition.

She scored a 27 out of 30 on mini mental status exam, losing two points in recall and one point in the three-stage

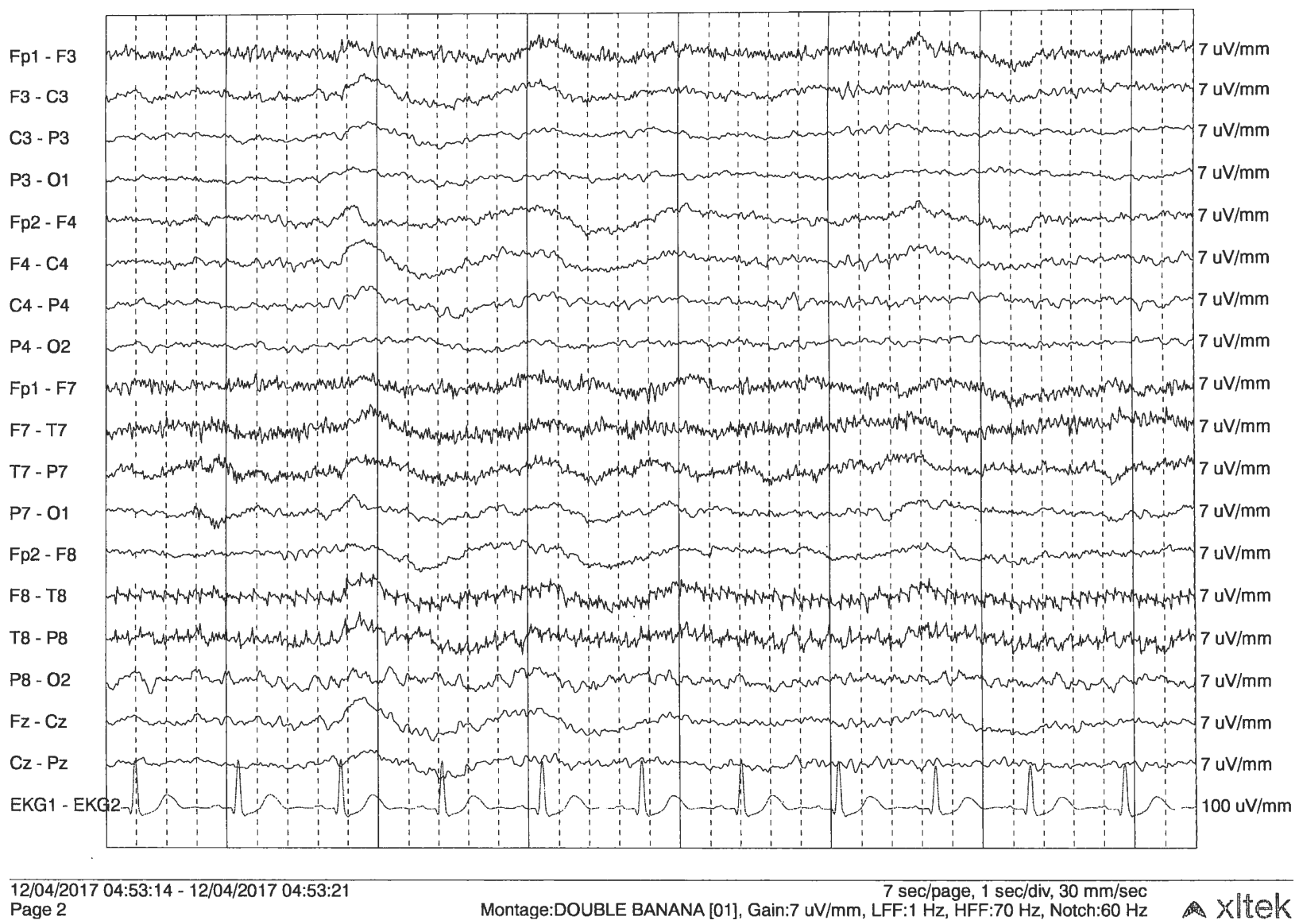

Figure I Continuous I7-hour video EEG monitoring.

Notes: The EEG was collected digitally and stored in a computer that has seizure detection as well as spike detection software. The patient can activate a push-button event as well as the nurse to indicate if a clinical event has occurred. With the patient awake, the background showed no clear dominant occipital rhythm over the left hemisphere. There was low voltage beta activity recorded diffusely. There is irregular polymorphic $\Delta / \theta$ activity recorded at 3-5 $\mathrm{Hz}$ maximal over the left temporoparietal region. Abbreviation: EEG, electroencephalogram. 
command. She was started on acamprosate for alcohol cravings and discharged to a subacute rehabilitation facility.

\section{Discussion}

Upon admission to the neurology intensive care unit, the medical team was managing a patient with a history of alcohol use disorder and epilepsy who was presenting at subtherapeutic phenytoin levels with altered mental status after throwing up for nearly 48 hours. During an admission 9 months earlier, she had been diagnosed with HSV encephalitis and possible WE syndrome. There was a high degree of suspicion for recurrent HSV infection, intermittent focal seizure activity, postictal psychosis, or pseudobulbar affect or a vascular cause of her fluent aphasia. There were no concerns for HE because the following: total bilirubin was 1.5 , patient was not jaundiced, no asterixis was present, and patient's confusion got better without any administration of lactulose. The absence of corresponding findings on MRI or EEG raised a concern for psychogenic etiology of aphasia, resulting in a psychiatric consult.

Postictal psychosis occurs almost exclusively in adults with chronic and treatment-refractory epilepsy, usually 15-20 years after their initial diagnosis affecting $25 \%-50 \%$ of this population. ${ }^{6}$ It presents with hallucinations and disorders of thought. Even though RR was confused, no hallucinations or delusions were present, reducing suspicion for this diagnosis.

Pseudobulbar affect commonly presents as emotional incontinence, or uncontrollable outbursts of laughter or crying at inappropriate times, and is found in patients with a history of brain injury or neurodegenerative disease. ${ }^{7}$ While RR demonstrated some disinhibition and laughed excessively, she had no history of neurodegenerative disease or brain damage and imaging was negative for corticobulbar tract degeneration.

Psychogenic aphasia, or conversion disorder, can be found in $4 \%-5 \%$ of adult patients with neurologic disorders. ${ }^{8}$ It is characterized by inconsistent mispronunciations and frequent stuttering. Unlike true aphasias, psychogenic aphasia worsens with time. RR's abnormal speech began suddenly, was fluent, and without stuttering.

Suspicion for recurrent HSV encephalitis was low, and our literature search of language changes in HSV encephalitis did not show evidence of fluent aphasias. A 2012 Brazilian case report details anomic aphasia, or the misidentification of objects using related words (saying "comb" instead of "hair") in a 13-year-old patient with herpes simplex encephalitis (HSE). ${ }^{9}$ Another case report from Italy notes a similar difficulty in naming objects in patients with HSE, particularly

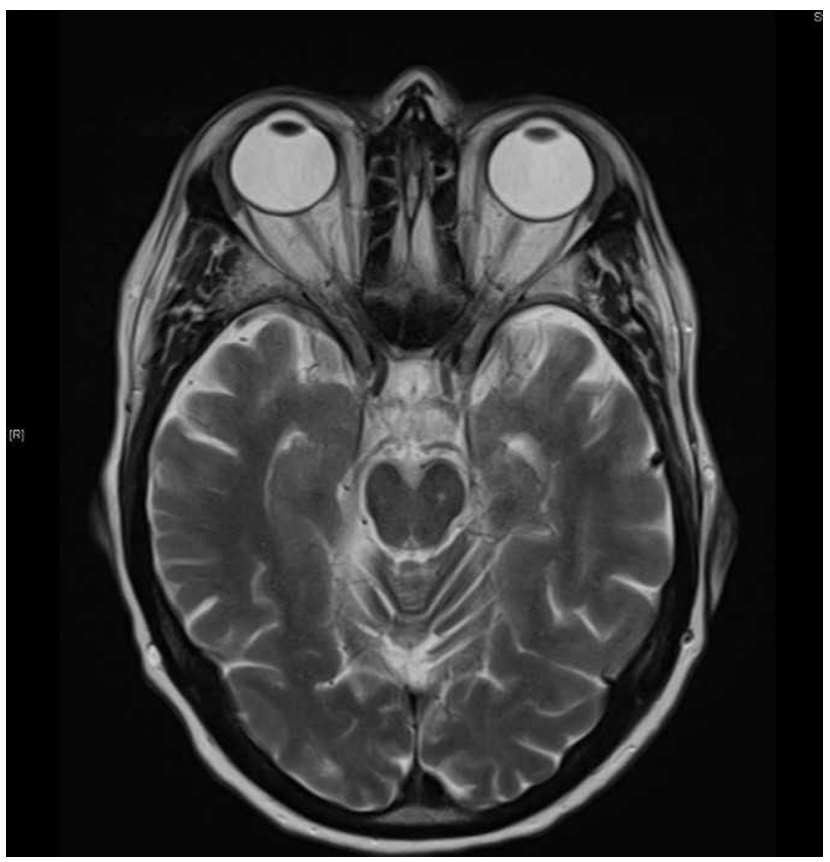

Figure 2 Current admission shows less pronounced left mesial temporal abnormality.

living objects (fruits, vegetables, and animals). ${ }^{10}$ None of the elements of our patient's exam are consistent with anomic aphasia. RR's most recent MRI (Figure 2) failed to show a temporal FLAIR abnormality; such a finding on a previous MRI (Figure 3) resulted in her initial HSV encephalitis diagnosis. It is possible however that the residual effects of her previous infection resulted in the temporal structural/ functional lesion found in the vEEG (Figure 1).

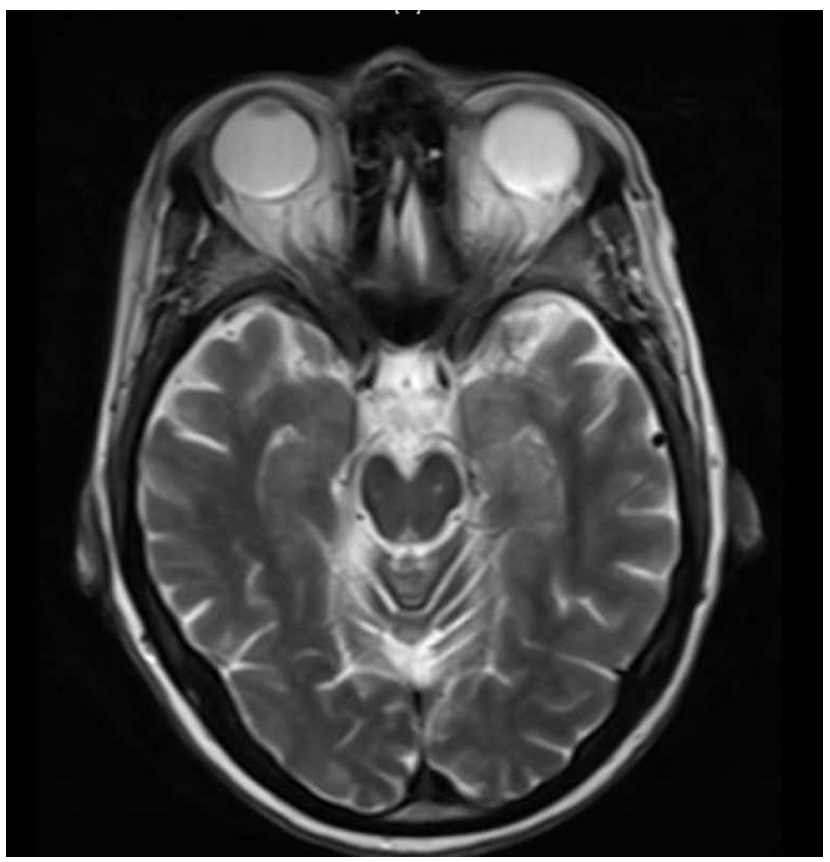

Figure 3 Admission 9 months prior showing nonspecific FLAIR abnormality in the left mesial temporal lobe. 
The focus now shifted to the patient's extensive alcohol abuse history. Fluent aphasia may be confused for confabulation, an unintentional fabrication of ideas and circumstances inconsistent with the truth. ${ }^{11}$ Confabulation is associated with permanent memory damage and can be seen in WK syndrome. Momentary or provoked confabulation is commonly seen in Korsakoff syndrome and is irreversible. ${ }^{12} \mathrm{Her}$ exam during hospitalization for suspected HSV encephalitis 9 months prior revealed spontaneous confabulation, causing the team at the time to believe she had WE. Spontaneous confabulation subsides in WE after clouding of consciousness subside. ${ }^{12}$ The patient's reversible language abnormality did not include the creation of narratives or stories but, rather, was a slew of disconnected words and phrases, more consistent with aphasia. Therefore, we do not believe that her presentation could be accounted for solely by her alcohol-induced WE.

Aphasias are commonly due to stroke, though trauma, infection, and malignancies have been known to produce them as well. ${ }^{13}$ While less common, postictal aphasias have been documented. Several case reports describe postictal motor and sensory aphasias, affecting language output and verbal memorization ability. ${ }^{14,15} \mathrm{~A}$ review of the literature revealed only one other case of a fluent aphasia in the postictal state: a 2012 British case report details a 60-year-old patient with sudden-onset fluent aphasia after prolonged status epilepticus, like our patient RR. ${ }^{5}$ This patient's aphasia was reversed in 3 days with levetiracetam $750 \mathrm{mg}$ BID and $500 \mathrm{mg}$ of intravenous thiamine every 8 hours; our patient's regimen varied only with the addition of aripiprazole $2.5 \mathrm{mg}$. The near-complete reversal of RR's aphasia prompted the question of what in her treatment regimen produced the return to baseline.

A partial dopamine agonist and antipsychotic, aripiprazole, has documented neuroprotective effects on animal models. A 2018 study redemonstrated previous studies' findings on aripiprazole, it is able to reduce cortical atrophy and restore motor function following ischemic attacks in mouse models. ${ }^{16}$ However, RR was on a low dose (2.5 mg). It was therefore the antiepileptic levetiracetam and its previously unexplored effect on aphasias and neuroprotection that gained the focus. The effect of the antiepileptic levetiracetam on reducing the severity of withdrawal-related GABA and glycine receptor hyperactivity is well summarized in a 2008 report. $^{17}$

Levetiracetam belongs to the racetam class of drugs, molecules with a pyrrolidone backbone, that also includes piracetam; the two are racemic structures and levetiracetam has an additional ethyl group. ${ }^{18}$ The neurorestorative properties of piracetam in patients with stroke-induced aphasia revealed a mild increase in "any language measure" over patients who did not take piracetam. ${ }^{19}$ A 2006 meta-analysis shed more light on its neurorestorative effects. A derivative of GABA, the molecule is believed to improve neuron membrane fluidity and therefore neurotransmission, thus acting as a restorative agent. ${ }^{20}$ This analysis also looked at a study that examined alcohol-related damage to phospholipid bilayers. Piracetam can confer protection in alcohol-induced stress by shielding the membrane from alcohol and increasing its fluidity, inducing individual molecules in the neuronal bilayer to reorganize. ${ }^{21}$ Given levetiracetam's similarity in structure to piracetam, its effects may have been to strengthen neuronal membranes in the temporal lobes, the areas hit by her seizures and a common location for alcohol-related brain changes. ${ }^{22}$ The patient was monitored on the CIWA protocol, receiving lorazepam $2 \mathrm{mg}$ once totally after CIWA was initiated. Levetiracetam's effect on alcohol withdrawal could explain the CIWA score decreasing with minimal benzodiazepine use.

RR's extensive history of alcohol dependence made us strongly consider withdrawal as the source of her seizures. Thiamine deficiency and alcohol withdrawal may have contributed to patient's vomiting. Seizures and delirium are complications of alcohol withdrawal, which also presents with language abnormalities such as slurred speech. Fluent aphasia has not been reported to be associated with acute withdrawal, and her vEEG changes suggested that an underlying lesion was the etiology of her seizures. Prior to the admission for encephalitis, she was seen in ER 1 year before for alcohol abuse and fall. After the admission for encephalitis and prior to the episode described in our manuscript, she was seen in ER four times, all for falls and related fractures. While many factors may have contributed to fall, WE is a strong possibility. Inconclusive MRI is a characteristic of WE. ${ }^{23}$ Aphasia was not identified during the previous visits. During the current admission, fluent aphasia was noted. It is possible the patient had WE at home, was confused, and was not able to care for herself and did not take Dilantin and then seized, followed by fluent aphasia which was observed in ER and hospital admission. We concluded that her seizures were likely the inciting factor of the patient's aphasia.

\section{Conclusion}

This patient's course was complicated by her history of epilepsy, alcohol abuse (with likely WE), and HSV encephalitis. We believe that her fluent aphasia was caused by her 
seizures. Her WE may have contributed to her acute altered mental status, and it almost certainly caused her occasional documented confabulation. The unique features of the following combination likely resulted in a reversal of RR's fluent aphasia: 1) high doses of levetiracetam, possibly conferring neuron membrane fluidity, 2) aripiprazole, a drug shown to halt brain atrophy in mouse models, and 3) a high dose $(1,500 \mathrm{mg})$ of parenteral thiamine to address her deficiency and likely WE. ${ }^{24}$ The administration of parenteral thiamine along with levetiracetam's effectiveness in reducing neuronal hyperactivity may have been instrumental in her improvement as an alcoholic patient. Levetiracetam should be considered in WE and the rare occurrence of aphasia after seizures.

\section{Disclosure}

The authors report no conflicts of interest in this work.

\section{References}

1. Victor M, Adams RD, Collins GH. The Wernicke-Korsakoff Syndrome. Philadelphia, PA: F. A. Davis; 1971.

2. Saad L, Silva LF, Banzato CE, Dantas CR, Garcia C. Anorexia nervosa and Wernicke-Korsakoff syndrome: a case report. J Med Case Rep. 2010;4:217.

3. Oudman E, van der Stigchel S, Postma A, Wijnia JW, Nijboer TC. A Case of Chronic Wernicke's Encephalopathy: A Neuropsychological Study. Front Psychiatry. 2014;5:59.

4. Caine D, Halliday GM, Kril JJ, Harper CG. Operational criteria for the classification of chronic alcoholics: identification of Wernicke's encephalopathy. J Neurol Neurosurg Psychiatry. 1997;62(1):51-60.

5. Patil B, Oware A. De-novo simple partial status epilepticus presenting as Wernicke's aphasia. Seizure. 2012;21(3):219-222.

6. Devinsky O, Common "postictal Psychosis: Dangerous, and Treatable.". Epilepsy Currents. 2008;8(2):31-34.

7. Ahmed A, Simmons Z. Pseudobulbar affect: prevalence and management. Ther Clin Risk Manag. 2013;9:483-489.

8. Binder LM, Spector J, Youngjohn JR. Psychogenic stuttering and other acquired nonorganic speech and language abnormalities. Arch Clin Neuropsychol. 2012;27(5):557-568.
9. Soares-Ishigaki EC, Cera ML, Pieri A, Ortiz KZ, Siqueira EC. Aphasia and herpes virus encephalitis: a case study. Sao Paulo Med J. 2012;130(5): 336-341.

10. Barbarotto R, Capitani E, Laiacona M, et al. Naming deficit in herpes simplex encephalitis. Acta Neurol Scand. 2009;93(4):272-280.

11. Trzepacz PT, Baker RW. The Psychiatric Mental Status Examination. Oxford: Oxford University Press; 1993.

12. Kopelman MD, Thomson AD, Guerrini I, Marshall EJ. The Korsakoff syndrome: clinical aspects, psychology and treatment. Alcohol Alcohol. 2009;44(2):148-154.

13. Goodglass H. Understanding Aphasia. San Diego: Academic Press; 1993:104.

14. Kudo T, Funakoshi A, Tanaka M, et al. Postictal aphasia and its generating mechanism in 3 patients with localization-related epilepsy. Seishin Shinkeigaku Zasshi. 1993;95(2):125-150.

15. Trebuchon A, Lambert I, Guisiano B, et al. The different patterns of seizure-induced aphasia in temporal lobe epilepsies. Epilepsy Behav. 2018;78:256-264.

16. Gil CH, Kim YR, Lee HJ, et al. Aripiprazole exerts a neuroprotective effect in mouse focal cerebral ischemia. Exp Ther Med. 2018;15(1): 745-750.

17. Sarid-Segal O, Piechniczek-Buczek J, Knapp C, et al. The effects of levetiracetam on alcohol consumption in alcohol-dependent subjects: an open label study. Am J Drug Alcohol Abuse. 2008;34(4):441-447.

18. Löscher W, Richter A. Piracetam and levetiracetam, two pyrrolidone derivatives, exert antidystonic activity in a hamster model of paroxysmal dystonia. Eur J Pharmacol. 2000;391(3):251-254.

19. Greener J, Enderby P, Whurr R. Pharmacological treatment for aphasia following stroke. Cochrane Database Syst Rev. 2001;(4):CD000424.

20. Winblad B. Piracetam: A Review of Pharmacological Properties and Clinical Uses. CNS Drug Rev. 2006;11(2):169-182.

21. Peuvot J, Schanck A, Deleers M, Brasseur R. Piracetam-induced changes to membrane physical properties. A combined approach by 31P nuclear magnetic resonance and conformational analysis. Biochem Pharmacol. 1995;50(8):1129-1134.

22. Tu X, Wang J, Liu X, Zheng J. Aberrant regional brain activities in alcohol dependence: a functional magnetic resonance imaging study. Neuropsychiatr Dis Treat. 2018;14:847-853.

23. Antunez E, Estruch R, Cardenal C, et al. Usefulness of CT and MR imaging in the diagnosis of acute Wernicke's encephalopathy. AJR Am J Roentgenol. 1998;171(4):1131-1137.

24. Thomson AD, Guerrini I, Marshall EJ. The evolution and treatment of Korsakoff's syndrome: out of sight, out of mind? Neuropsychol Rev. 2012;22(2):81-92.
Neuropsychiatric Disease and Treatment

\section{Publish your work in this journal}

Neuropsychiatric Disease and Treatment is an international, peerreviewed journal of clinical therapeutics and pharmacology focusing on concise rapid reporting of clinical or pre-clinical studies on a range of neuropsychiatric and neurological disorders. This journal is indexed on PubMed Central, the 'PsycINFO' database and CAS,

\section{Dovepress}

and is the official journal of The International Neuropsychiatric Association (INA). The manuscript management system is completely online and includes a very quick and fair peer-review system, which is all easy to use. Visit http://www.dovepress.com/testimonials.php to read real quotes from published authors. 